\title{
Influence of Two Photon Absorption on Soliton Self-Frequency Shift
}

\author{
Steffensen, Henrik; Rottwitt, Karsten; Jepsen, Peter Uhd; Bang, Ole
}

Published in:

Proceedings of Nonlinear Optics: Materials, Fundamentals and Applications (NLO)

Publication date:

2011

Document Version

Publisher's PDF, also known as Version of record

Link back to DTU Orbit

Citation (APA):

Steffensen, H., Rottwitt, K., Jepsen, P. U., \& Bang, O. (2011). Influence of Two Photon Absorption on Soliton Self-Frequency Shift. In Proceedings of Nonlinear Optics: Materials, Fundamentals and Applications (NLO) (pp. NWE16)

\section{General rights}

Copyright and moral rights for the publications made accessible in the public portal are retained by the authors and/or other copyright owners and it is a condition of accessing publications that users recognise and abide by the legal requirements associated with these rights.

- Users may download and print one copy of any publication from the public portal for the purpose of private study or research.

- You may not further distribute the material or use it for any profit-making activity or commercial gain

- You may freely distribute the URL identifying the publication in the public portal

If you believe that this document breaches copyright please contact us providing details, and we will remove access to the work immediately and investigate your claim. 


\title{
Influence of Two Photon Absorption on Soliton Self-Frequency Shift
}

\author{
H. Steffensen, K. Rottwitt, P. U. Jepsen, and O. Bang \\ DTU Fotonik, Technical University of Denmark, Ørsteds Plads 343, DK-2800 Kongens Lyngby, Denmark \\ Tel: +4545256352 Fax: +4545936581 \\ hste@fotonik.dtu.dk
}

\begin{abstract}
The creation of mid-infrared supercontinua necessitates the use of soft-glass fibers. However, some materials, like chalcogenide, have a substantial two photon absorption. We introduce a model for soliton self-frequency shift that successfully includes this effect.
\end{abstract}

(C) 2011 Optical Society of America

OCIS codes: $060.4370,060.5530$.

\section{Introduction}

The fundamentals in achieving a wide supercontinuum spectrum is strong pumping in an optical fiber close to the zero dispersion point to create soliton fission, which allows for fundamental solitons to self-frequency shifting to form the red edge of the spectrum, while group velocity matched dispersive waves blue shift to form the blue edge [1]. Due to the loss edge of silica, it is not possible to extend the supercontinuum into the mid-infrared. Instead there is an increasing interest in using soft-glass fibers such as chalcogenide [2,3] for mid-infrared supercontinuum generation (SCG). However in chalcogenide as an example, there is a high level of two photon absorption (TPA) [4], which causes a reduction of peak power and thus leads to a reduced soliton self-frequency shift (SSFS). Existing models [5-7] do not account for this, so we derive a new model which for the first time accounts for the influence of TPA on the SSFS.

\section{Model}

The model is based on the generalized nonlinear Schrödinger equation (GNLS), as given by

$$
\partial_{z} u(z, t)+\frac{\alpha}{2} u(z, t)-i \sum_{m \geq 2} \frac{i^{m} \beta_{m}}{m !} \partial_{t}^{m} u(z, t)=i \sum_{n \geq 0} \frac{i^{n} \gamma_{n}}{n !} \partial_{t}^{n}\left[u(z, t) \int R\left(t^{\prime}\right)\left|u\left(z, t-t^{\prime}\right)\right|^{2} \mathrm{~d} t^{\prime}\right],
$$

where $u$ is the complex field envelope at carrier frequency $\omega_{0}, \alpha$ is linear loss, $\beta_{m}$ and $\gamma_{m}$ are the $m$ 'th derivative at $\omega_{0}$ of the group velocity dispersion (GVD) and the nonlinear coefficient, respectively, and finally $R(t)$ is the time response given by $R(t)=\left(1-f_{R}\right) \delta(t)+f_{R} h_{R}(t)$, which includes both the instantaneous Kerr and delayed Raman response, given as $h_{R}(t)$. The nonlinear coefficient, when TPA is present, is given by [8]

$$
\gamma=\frac{2 \pi n_{2}}{\omega A_{\mathrm{eff}}}+\frac{i \beta_{\mathrm{TPA}}}{2 A_{\mathrm{eff}}}
$$

where $n_{2}$ is the nonlinear refractive index, $c$ is the speed of light, $\beta_{\text {TPA }}$ is the TPA coefficient and $A_{\text {eff }}$ is the effective area. Only the first two elements of each sum have been included. The model is based on the soliton ansatz

$$
u(z, t)=\sqrt{P_{0}(z)} \operatorname{sech}\left(\frac{t-t_{c}(z)}{T_{0}(z)}\right) \exp \left[i \Phi(z)-i b(z)\left(t-t_{c}(z)\right)-i \mu(z)\left(t-t_{c}(z)\right)^{2}\right]
$$

with $b, t_{c}, T_{0}, P_{0}, \mu$ being the frequency shift, pulse center, width, peak power, and quadratic chirp, respectively. With this ansatz and the method of moments, based on the evolution of five moments, it is possible to derive a set of differential equation for the soliton parameters $b, t_{c}, T_{0}, P_{0}, \mu[6,7]$. In a moving frame of reference and for long pulses, 
the equations reduce to

$$
\begin{aligned}
& \frac{d b}{d z} \approx-\frac{8}{15} \frac{\gamma_{0}^{r} P_{0} T_{R}}{T_{0}^{2}}+\frac{4}{3} \mu \gamma_{1}^{r} P_{0}-\frac{4}{3} \mu \gamma_{0}^{i} P_{0} T_{R}-\frac{4}{5} \frac{\gamma_{1}^{i} P_{0}}{T_{0}^{2}}-4\left(\frac{\pi^{2}}{9}-\frac{2}{3}\right) \mu^{2} \gamma_{1}^{i} P_{0} T_{0}^{2}, \\
& \frac{d t_{c}}{d z} \approx \frac{1}{2} \beta_{3}\left[\frac{\pi^{2}}{3} \mu^{2} T_{0}^{2}+\frac{1}{3 T_{0}^{2}}\right]+\gamma_{1}^{r} P_{0}-\frac{2}{3} \gamma_{0}^{i} P_{0} T_{R}-2\left(\frac{\pi^{2}}{9}-\frac{2}{3}\right) \mu \gamma_{1}^{i} P_{0} T_{0}^{2}, \\
& \frac{d T_{0}}{d z} \approx 2 \mu T_{0} \beta_{2}+\frac{8}{\pi^{2}} \frac{\gamma_{1}^{r} P_{0} T_{R}}{T_{0}}+\frac{4}{\pi^{2}} \gamma_{0}^{i} P_{0} T_{0}-\left(\frac{4}{3}-\frac{12}{\pi^{2}}\right) \mu \gamma_{1}^{i} P_{0} T_{0} T_{R}, \\
& \frac{d P_{0}}{d z} \approx-\alpha P_{0}-2 \mu P_{0} \beta_{2}-\left(\frac{8}{\pi^{2}}+\frac{8}{15}\right) \frac{\gamma_{1}^{r} P_{0}^{2} T_{R}}{T_{0}^{2}}-\left(\frac{4}{3}+\frac{4}{\pi^{2}}\right) \gamma_{0}^{i} P_{0}^{2}-\frac{12}{\pi^{2}} \mu \gamma_{1}^{i} P_{0}^{2} T_{R}, \\
& \frac{d \mu}{d z} \approx\left(\frac{2}{\pi^{2}} \frac{1}{T_{0}^{4}}-2 \mu^{2}\right) \beta_{2}+\frac{2}{\pi^{2}} \frac{\gamma_{0}^{r} P_{0}}{T_{0}^{2}}-\left(\frac{8}{15}-\frac{4}{\pi^{2}}\right) \frac{\mu \gamma_{1}^{r} P_{0} T_{R}}{T_{0}^{2}}-\frac{76}{15 \pi^{2}} \frac{\gamma_{1}^{i} P_{0} T_{R}}{T_{0}^{4}},
\end{aligned}
$$

where the superscript on $\gamma_{n}^{r}$ and $\gamma_{n}^{i}$ refer to the real and imaginary part, respectively. The full version of the equations, valid for all pulse widths, are too complicated to include in this summary. By looking only on linear loss and loss directly caused by TPA in Eg. (4d), it is possible to obtain an analytical expression for the TPA loss length,

$$
L_{\mathrm{TPA}}=\frac{1}{\alpha} \ln \left[\frac{e+\left(\frac{4}{3}+\frac{4}{\pi^{2}}\right) \frac{\gamma_{0}^{i} P_{0}}{\alpha}}{1+\left(\frac{4}{3}+\frac{4}{\pi^{2}}\right) \frac{\gamma_{0}^{i} P_{0}}{\alpha}}\right]
$$

which is defined as the length of propagation after which the power is reduced to 1/e of its original value. After this length of propagation, the power has been reduced to such an extent as to effectively halt red-shifting.

\section{SSFS in Chalcogenide Fiber}

To demonstrate the effect of TPA, a $\mathrm{As}_{2} \mathrm{Se}_{3}$ chalcogenide fiber has been used for the simulations. The results of the analytic model has been compared to simulations of the GNLS equation to demonstrate the validity. The parameters are for a realistic fiber used for SCG. From Yeom et. al. [2] we have $\alpha \approx 0.5 \mathrm{~dB} / \mathrm{m}$ and $\beta_{2}=-360 \mathrm{ps}^{2} / \mathrm{km}, \beta_{3}=$ $3.58 \mathrm{ps}^{3} / \mathrm{km}$ and $A_{\text {eff }}=0.4773 \mu \mathrm{m}^{2}$ at $1550 \mathrm{~nm}$. The time response have been approximated as a decaying harmonic oscillator with $\tau_{1}=23$ fs and $\tau_{2}=210$ fs and $f_{R}=0.1$ based on $\mathrm{Hu}$ et. al. [3] and finally we have the nonlinear parameters in Table 1 from Nguyen et. al. [4]. The figure of merit (FOM) is defined as FOM $=n_{2} /\left(\lambda \beta_{\text {TPA }}\right)$

Table 1. Used values of the nonlinearity and two photon absorption

\begin{tabular}{rrl}
\hline$\lambda(\mathrm{nm})$ & $n_{2}\left(10^{-18} \mathrm{~m}^{2} / \mathrm{W}\right)$ & $\mathrm{FOM}$ \\
\hline 1415 & 11.0 & 0.8 \\
1434 & 14.0 & 0.9 \\
1456 & 13.0 & 1.1 \\
1491 & 9.9 & 1.45 \\
1515 & 8.9 & 1.8 \\
1554 & 7.0 & 1.8 \\
\hline
\end{tabular}

The results in Fig. 1 show that TPA has a clear effect in reducing the red shifting of a soliton, for a soliton with an initial width of $25 \mathrm{fs}$, the total red shift is reduced from $27 \mathrm{~nm}$ to $17 \mathrm{~nm}$ predicted by the proposed model and from $30 \mathrm{~nm}$ to $17 \mathrm{~nm}$ by simulation of the GNLS. The reduction in SSFS is predominantly caused by the reduction in peak power caused by the TPA. It also shows that the model in a qualitative manner shows what occurs in SSFS both with and without including TPA. For the short pulse, a reverse frequency shift is seen to occur around $z=4 \mathrm{~cm}$ and $13.5 \mathrm{~cm}$, caused by the second term in Eq. (4a) and a combination of a broadening of the pulse, a positive chirp and a relatively large $\gamma_{1}^{r}$, roughly an order of magnitude higher than the usual approximation of $\gamma_{0}^{r} / \omega_{0}$. It is an effect that will be further investigated in future work. 


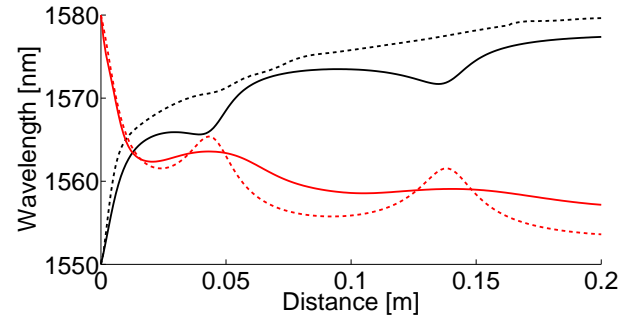

(a) $T_{0}=25 \mathrm{fs}$ where TPA is neglected.

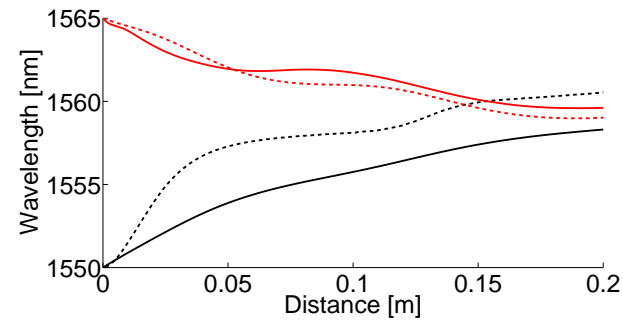

(c) $T_{0}=50 \mathrm{fs}$ where TPA is neglected.

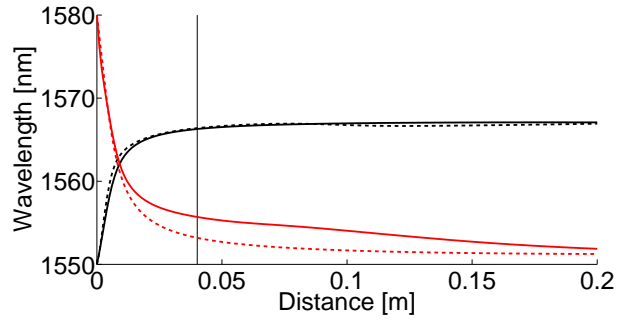

(b) $T_{0}=25$ fs with TPA included.

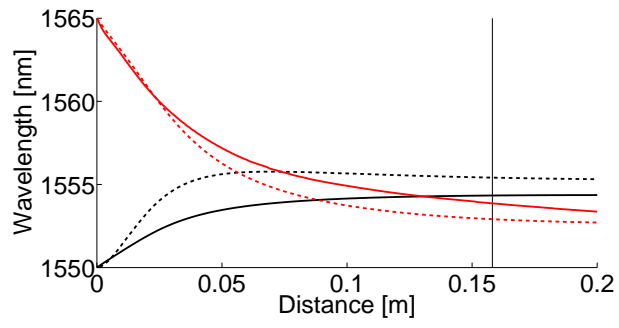

(d) $T_{0}=50$ fs with TPA included.

Fig. 1. Soliton dynamics when neglecting (a), (c) and including (b), (d) TPA for a fundamental soliton with a width of $25 \mathrm{fs}$ (a), (b) and $50 \mathrm{fs}$ (c), (d). The black lines (all originating in the lower left corner) displays the redshift while the red lines (originating in the upper left corner) show the normalized peak power of the soliton. The solid line is obtained with the proposed model while the dashed is from a full simulation of the GNLS. In the two cases where TPA is included, the theoretical TPA loss length have been marked by a vertical line.

\section{Conclusion}

In summary we have presented a model that successfully account for SSFS under the influence of TPA. Furthermore, it has incorporated the full Raman spectrum and is thus valid for solitons of any width. It accurately depicts the decrease in SSFS caused by TPA in a realistic chalcogenide fiber, specifically for a soliton with a width of 25 fs the total redshift is reduced by $10 \mathrm{~nm}$ from $27 \mathrm{~nm}$ to $17 \mathrm{~nm}$.

\section{References}

1. J. M. Dudley, G. Genty, and S. Coen, "Supercontinuum generation in photonic crystal fiber," Reviews of Modern Physics 78, 1135-1184 (2006).

2. D.-I. Yeom, E. C. Mägi, M. R. E. Lamont, M. A. F. Roelens, L. Fu, and B. J. Eggleton, "Low-threshold supercontinuum generation in highly nonlinear chalcogenide nanowires," Opt. Lett. 33, 660-662 (2008).

3. J. Hu, C. R. Menyuk, L. B. Shaw, J. S. Sanghera, and I. D. Aggarwal, "Maximizing the bandwidth of supercontinuum generation in $\mathrm{As}_{2} \mathrm{Se}_{3}$ chalcogenide fibers," Opt. Express 18, 6722-6739 (2010).

4. H. Nguyen, K. Finsterbusch, D. Moss, and B. Eggleton, "Dispersion in nonlinear figure of merit of $\mathrm{As}_{2} \mathrm{Se}_{3}$ chalcogenide fibre," Electron. Lett. 42, 20060605 (2006).

5. A. C. Judge, O. Bang, B. J. Eggleton, B. T. Kuhlmey, E. C. Mägi, R. Pant, and C. M. de Sterke, “Optimization of the soliton self-frequency shift in a tapered photonic crystal fiber," J. Opt. Soc. Am. B 26, 2064-2071 (2009).

6. E. N. Tsoy and C. M. de Sterke, "Dynamics of ultrashort pulses near zero dispersion wavelength," J. Opt. Soc. Am. B 23, 2425-2433 (2006).

7. Z. Chen, A. J. Taylor, and A. Efimov, "Soliton dynamics in non-uniform fiber tapers: analytical description through an improved moment method," J. Opt. Soc. Am. B 27, 1022-1030 (2010).

8. L. Yin, Q. Lin, and G. P. Agrawal, "Soliton fission and supercontinuum generation in silicon waveguides," Opt. Lett. 32, 391-393 (2007). 